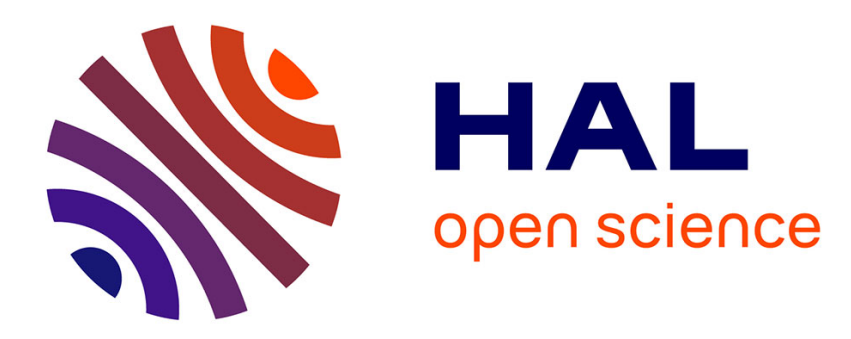

\title{
Obtention de la phase orthorhombique dans l'alliage Ti52Al30Nb18
}

S. Lokmane, M. Guymont

\section{To cite this version:}

S. Lokmane, M. Guymont. Obtention de la phase orthorhombique dans l'alliage Ti52Al30Nb18. Journal de Physique IV Proceedings, 1996, 06 (C2), pp.C2-59-C2-64. 10.1051/jp4:1996208 . jpa00254186

\section{HAL Id: jpa-00254186 https://hal.science/jpa-00254186}

Submitted on 1 Jan 1996

HAL is a multi-disciplinary open access archive for the deposit and dissemination of scientific research documents, whether they are published or not. The documents may come from teaching and research institutions in France or abroad, or from public or private research centers.
L'archive ouverte pluridisciplinaire HAL, est destinée au dépôt et à la diffusion de documents scientifiques de niveau recherche, publiés ou non, émanant des établissements d'enseignement et de recherche français ou étrangers, des laboratoires publics ou privés. 


\title{
Obtention de la phase orthorhombique dans l'alliage $\mathbf{T i}_{52} \mathbf{A l}_{\mathbf{3 0}} \mathbf{N b}_{\mathbf{1 8}}$
}

S. Lokmane* et M. Guymont*,**

* Centre d'Etude de Chimie Métallurgique, CNRS, 15 rue Georges Urbain, 94407 Vitry-sur-Seine cedex, France

** Laboratoire de Métallurgie Structurale, Université de Paris XI, Bât. 413, 91405 Orsay cedex, France

\begin{abstract}
Several phases may appear in the ternary $\mathrm{Ti}-\mathrm{Al}-\mathrm{Nb}$, among others : cubic $\beta$, hexagonal $\alpha$, and (recently identified) orthorhombic $O$. The special composition $\mathrm{Ti}_{52} \mathrm{Al}_{30} \mathrm{Nb}_{18}$ has lead to one-phased orthorhombic through adequate thermal treatment. The results of observations using $\mathrm{X}$-ray diffraction, transmission and scanning electron microscopy, are shown. The untreated samples are mainly B2; the samples annealed at $1100^{\circ} \mathrm{C}$ or $900^{\circ} \mathrm{C}$, and subsequently quenched, are many-phased $\alpha 2+\mathrm{O}+$ possibly B2; the long annealed $(340 \mathrm{~h}$; $900^{\circ} \mathrm{C}$ ) samples are completely orthorhombic.

This final state $\mathrm{O}$ seems to be reached, both through a massive transformation $\mathrm{B} 2 \rightarrow \mathrm{O}$ and an ordering $\alpha 2 \rightarrow \mathrm{O}$.
\end{abstract}

\section{INTRODUCTION}

Les alliages à base Ti-Al présentent, de par leur faible densité et leur bonne tenue mécanique, un grand intérêt pour l'aéronautique. Leur ductilité à haute température peut être améliorée par des additions de $\mathrm{Nb}, \mathrm{V}$ ou Mo [1]. Nous nous sommes intéressés au ternaire Ti-Al-Nb.

Plusieurs phases peuvent y apparaître: la phase $\beta$ cubique (A2) et sa forme ordonnée $\mathrm{B} 2$ ( $\mathrm{CsCl}$ ), une phase hexagonale $\alpha_{2}$ de structure $\mathrm{DO}_{19}\left(\mathrm{P}_{3} / \mathrm{mmc}\right)$ et une phase orthorhombique $\mathrm{O}(\mathrm{Cmcm})$, mise en évidence par Banerjee et al en 1988 [2].

Notre étude a pour but d'obtenir un alliage orthorhombique monophasé. Les données actuelles [3] nous ont incité à choisir la composition $\mathrm{Ti}_{52} \mathrm{AL}_{30} \mathrm{Nb}_{18}$.

\section{PROCEDURE EXPERIMENTALE}

\subsection{Elaboration de l'alliage}

L'élaboration a été effectuée en trois étapes :

1) Préparation du binaire $\mathrm{Ti}-\mathrm{Nb}$ dans un four à induction sur nacelle de cuivre refroidie.

2) Adjonction d'aluminium suivie de plusieurs refusions au four à induction.

3) Nouvelle fusion de l'ensemble dans un four à lévitation pour homogénéisation.

L'analyse chimique par spectrométrie d'émission plasma (I.C.P) et l'analyse $\mathrm{X}$ à dispersion d'énergie (E.D.X) montrent que l'échantillon est homogène et possède la bonne composition. 


\subsection{Traitements thermiques}

Différents traitements thermiques en ampoules scellées ont été effectués sur les échantillons (Cf. Tableau 1).

On notera que tous les traitements thermiques à $900^{\circ} \mathrm{C}$ sont précédés d'un recuit à $1100^{\circ} \mathrm{C}$ pendant $3 \mathrm{~h}$, suivi d'un refroidissement dans l'eau sans casser l'ampoule.

\section{RESULTATS}

Les techniques de caractérisation utilisées sont la diffraction des rayons $\mathrm{X}$ (anticathode de cobalt), la diffraction et la microscopie électronique en transmission (MET), la microscopie électronique à balayage (MEB) et l'analyse X en dispersion d'énergie.couplée au MEB.

\subsection{Echantillon brut (monophasé B2):}

L'échantillon brut d'élaboration est principalement monophasé B2 (Fig 1), avec néanmoins des pics de la phase orthorhombique de très faible intensité qui témoignent de l'instabilité de B2.

Les observations au MET à grandissement moyen font apparaître un aspect "tweed" qui se traduit en diffraction, par de la diffusion suivant $\{112\}$ (Fig.2).

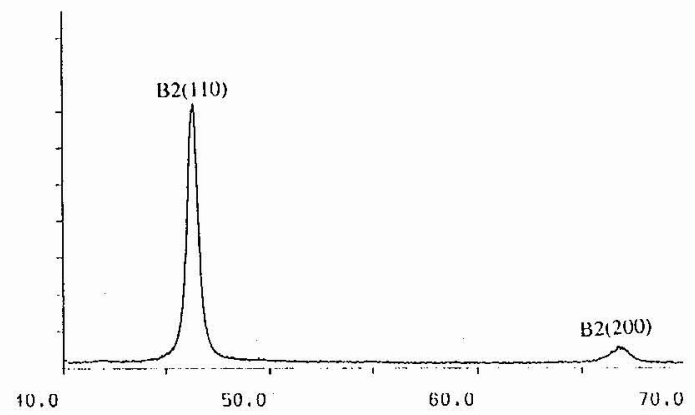

Figure 1: Spectre de diffraction $\mathrm{X}$ d'un échantillon à l'état brut.

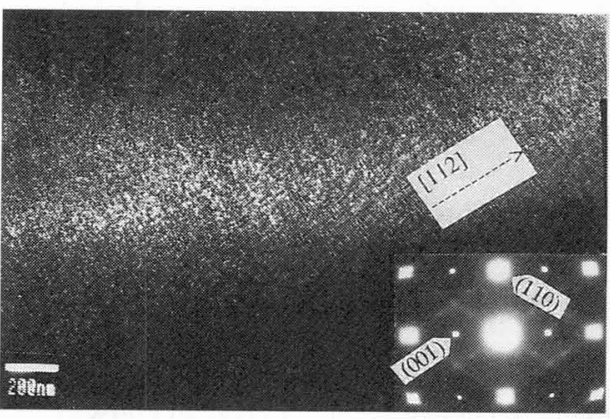

Figure 2: Image en champ clair (MET) et cliché de diffraction électronique de l'échantillon à l'état brut.

\subsection{Echantillon 1 (biphasé $\alpha_{2}+$ B2):}

L'échantillon 1 est biphasé $\left(\alpha_{2}+\right.$ B2), comme le montre le spectre de diffraction X (Fig.3). Ceci est confirmé par l'image au MET sur laquelle on voit des domaines de $\alpha 2$ dans une matrice B2 (Fig.4).

L'analyse $X$ en dispersion d'énergie montre que la phase $\alpha 2$ est saturée en nobium, avec une teneur de $12,5 \%$ atomique. Il semblerait que cette phase apparaisse préférentiellement sur les joints de grains de la phase B2.

A l'intérieur des grains, la phase $\alpha_{2}$ se présente sous forme de domaines allongés parallèles, dont la densité de répartition diminue en allant de la périphérie, vers le coeur de l'échantillon. 


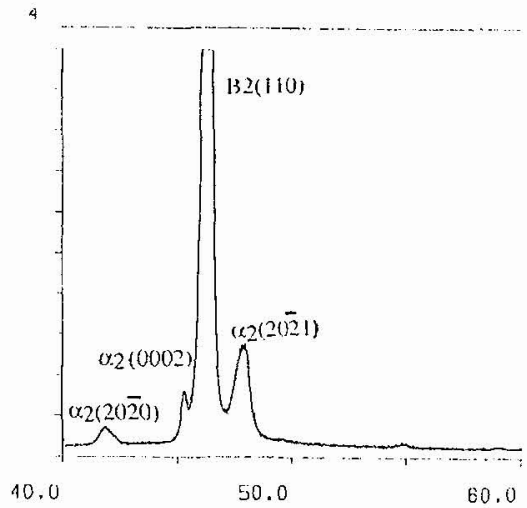

Figure 3: Spectre de diffraction $\mathrm{X}$ de $\mathrm{l}^{\prime}$ échantillon 1.

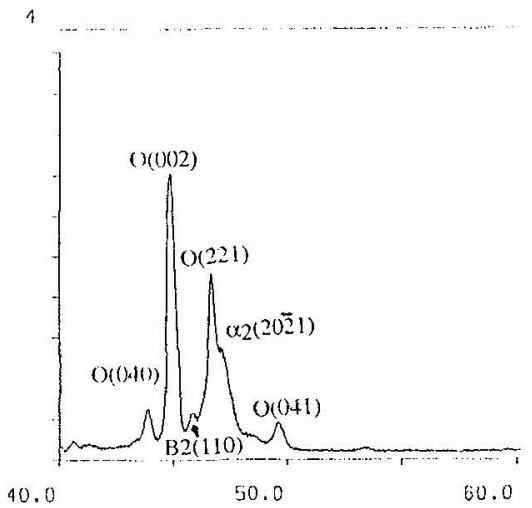

Figure 5: Spectre de diffraction $\mathrm{X}$ de $\mathrm{I}$ échantillon 5.

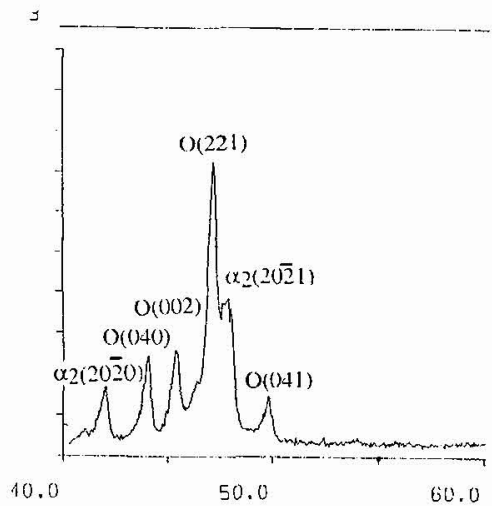

Figure 7: Spectre de diffraction $\mathrm{X}$ de l' échantillon 2.

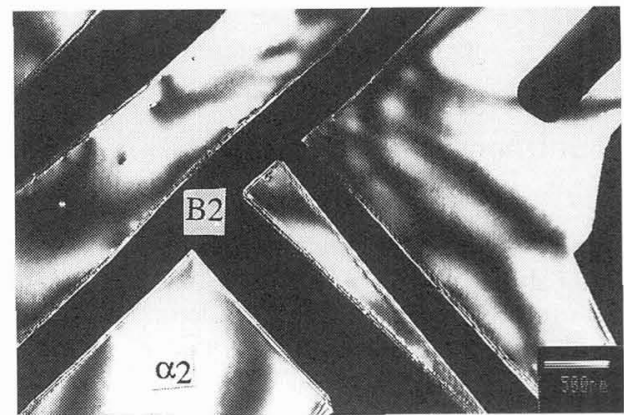

Figure 4: Image en champ clair (MET) de l'échantillon 1.

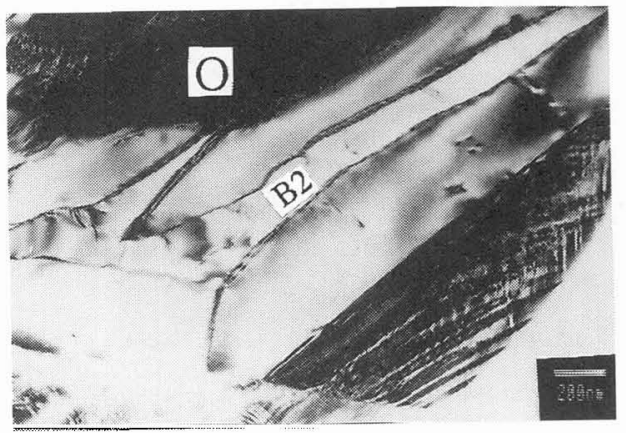

Figure 6: Image en champ clair (MET) de l'échantillon 5

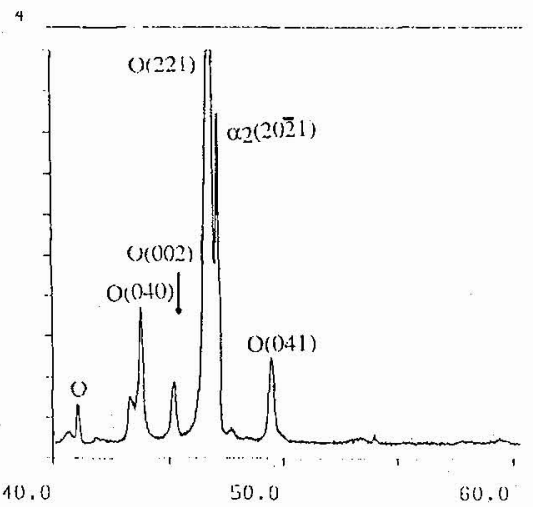

Figure 8: Spectre de diffraction $\mathrm{X}$ de l' échantillon 3. 


\subsection{Echantillon 5 (état triphasé $O+\alpha_{2}+B 2$ résiduel):}

L'échantillon 5, refroidi plus lentement (four coupé), présente les trois phases : $\mathrm{O}, \alpha_{2}$, et des résidus de B2 (Fig.5 et 6).

\subsection{Echantillons 2 et 3 (polyphasés):}

Ces échantillons ont tous subi les traitements A puis B, pendant différentes durées, suivis d'une trempe à l'eau (Cf. Tableau.1).

Après 30 minutes de recuit à $900^{\circ} \mathrm{C}$, la phase $\mathrm{B} 2$ se transforme majoritairement en $\mathrm{O}$. L'échantillon 2 est constitué de $\alpha_{2}$ initial, de O provenant de B2, et de B2 résiduel (Fig.7). Si on augmente le temps de recuit jusqu'à 100 heures, le résidu B2 disparaît totalement (Fig.8). L'observation au MEB montre par ailleurs une autre phase très riche en niobium $(\approx 30 \%$ at de $\mathrm{Nb}$ ) qui précipite dans $\alpha 2$ (Fig.9).

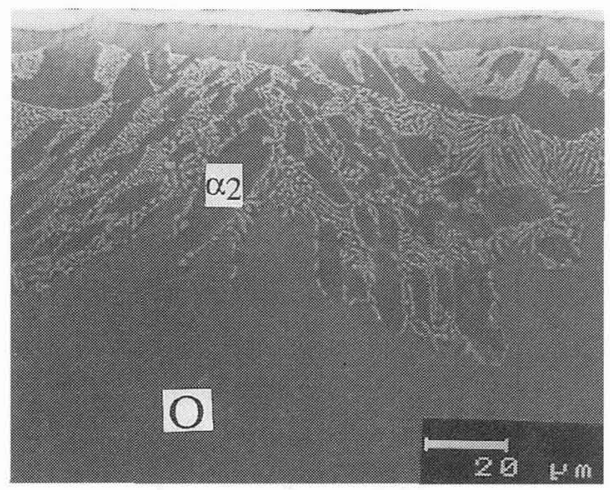

Figure 9: Image au MEB de l'échantillon 3.

\subsection{Echantillon 4 (monophasé 0 ):}

Cet échantillon, recuit pendant 340 heures, est totalement orthorhombique (Fig. 10,11).

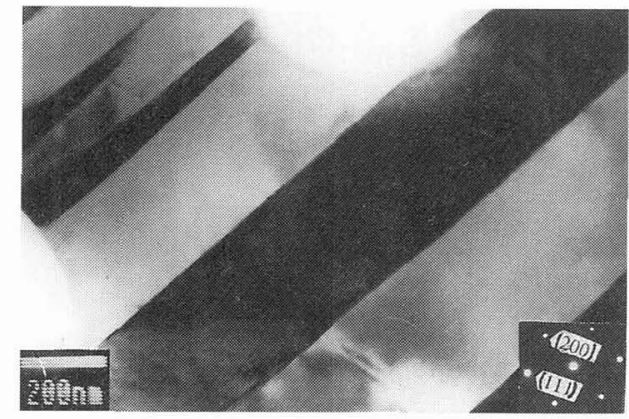

Figure 10: Image en champ clair (MET) de l'échantillon 4

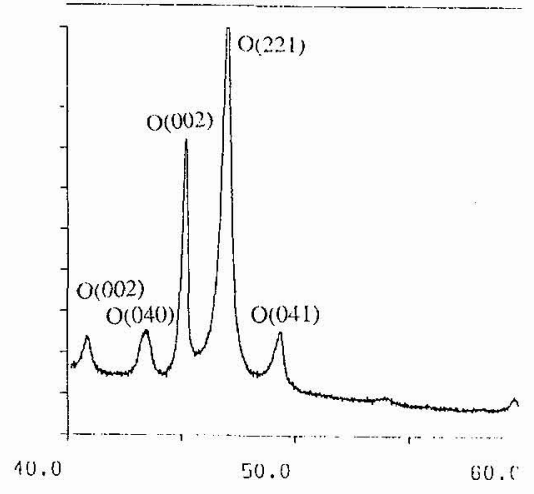

Figure 11: Spectre de diffraction X de l' échantillon 4. 


\section{Discussion:}

L'alliage $\mathrm{Ti}_{52} \mathrm{Al}_{30} \mathrm{Nb}_{18}$ à l'état brut d'élaboration est complètement ordonné $\mathrm{B} 2$; aucune paroi d'antiphase n'a été observée. Cependant, les réflexions de surstructures B2 observées en diffraction électronique, sont invisibles sur le spectre de diffraction X. Il semble que leur intensité soit très faible par rapport à l'intensité des pics fondamentaux, ce qui confirme les travaux de Hsiung [4].

\subsection{Transformation $B 2$-...-> $\alpha_{2}$ :}

Un recuit à $1100^{\circ} \mathrm{C}$ suivi d'un refroidissement à l'eau, transforme une partie de B2 en $\alpha_{2}$. Cette transformation s'effectue par germination et croissance de $\alpha_{2}$ préférentiellement sur les joints de grains de $\mathrm{B} 2$, mais aussi à l'intérieur des grains.

La phase $\alpha_{2}$ étant saturée au maximum à $13 \%$ de niobium [5]. la transformation B2 --> $\alpha_{2}$, pour notre composition (18\% at. de $\mathrm{Nb}$ ), est contrôlée par la diffusion anti-Fick du niobium.

\subsection{Transformation $\mathrm{B} 2 \quad \cdots . . .>\mathrm{O}$ :}

Le chauffage à $900^{\circ} \mathrm{C}$ de l'échantillon 1 , constitué de $\left(\mathrm{B} 2+\alpha_{2}\right)$, provoque la transformation massive de B2 en $\mathrm{O}$ [6-7]. Cette transformation se fait sans changement de composition, avec la relation d'orientation suivante entre la phase mère et la phase fille:

$$
(001)_{\mathrm{O}} / /(110)_{\mathrm{B} 2} \quad \text { et } \quad[110]_{\mathrm{O}} / /[111]_{\mathrm{B} 2}
$$

\subsection{Transformation $\alpha 2 \quad \cdots-->0$ :}

La transformation de $\alpha_{2}$ en $\mathrm{O}$ à $900^{\circ} \mathrm{C}$ se fait lentement [8]. Pour notre composition, la transformation n'est complète qu'après 340 heures.

Comme l'ont montré Banerjee et Muraleedharan [9], les plans $(0001)_{\alpha 2}$ et $(001)_{O}$ sont semblables dans les deux structures. La transformation de $\alpha_{2}$ en $\mathrm{O}$ se fait alors par une légère distorsion, accompagnée d'un faible changement de composition.

\section{Conclusion:}

La transformation de $\mathrm{B} 2$ en $\mathrm{O}$ peut se faire par 2 chemins différents:

1 - Chemin direct: B2 se transforme massivement en $\mathrm{O}$ sans changement de composition.

2 - Chemin indirect: B2 se transforme tout d'abord par germination et croissance en $\alpha 2$, donnant ensuite $\mathrm{O}$; la cinétique de cette deuxième transformation est très lente. 


\begin{tabular}{|c|c|c|c|c|}
\hline Echantillons & Température & Temps & $\begin{array}{c}\text { Mode de } \\
\text { refroidissement }\end{array}$ & Phases présentes \\
\hline 1 & $1100^{\circ} \mathrm{C}$ & $3 \mathrm{~h}$ & $\begin{array}{l}\text { dans l'eau (sans } \\
\text { casser l'ampoule) }\end{array}$ & $\mathrm{B} 2+\alpha_{2}$ \\
\hline \multicolumn{5}{|c|}{ TRAITEMENT B } \\
\hline 3
\end{tabular}

Tableau 1: Ensemble des traitements thermiques effectués et des phases obtenues.

\section{References}

[1] Ward C. H., Inter. Mater. Rev. 38(1993) 79-101.

[2] Banerjee D., Gogia A. K., Nandy T. K. et Joshi V.A., Acta. Metall., 36(1988) 871-882.

[3] Rowe R. G., Banerjee D., Muraleedharan K., Larsen M., Hall E., L., Konitzer D. G. et Woodfield, A. P., Titanium '92 Science and Technology, 1993., F. H. Froes and I. L. Caplan Eds. (Warrandale, Pennsylvania: Metallurgical Society of AIME), p. 1259.

[4] Hsiung L. M. and Wadley H. N. G., Mater. Sci. Eng. A 192/193(1995) 908-913.

[5] Muraleedharan K., Nandy T. K. Banerjee D. et Lele S., Intermetallics 3 (1995) 187-199.

[6] Kestner- Weykamp H. T., Ward C. H., Broderick T. F. et Kaufman M. J., Scripta Met. 23(1989) 1697-1702.

[7] Weykamp H. T., Baker D. R., Paxton D. M. and Kaufman M. J., Scripta Metall. Mater 24(1990) 445450.

[8] Muraleedharan K., Banerjee D., Scripta Metall. Mater 29(1993) 527.

[9] Muraleedharan K., Banerjee D., Philos. Mag. A 71(1995) 1011-1036. 